\title{
Germline contamination and leakage in whole genome somatic single nucleotide variant detection
}

\author{
Dorota H. Sendorek ${ }^{1 \dagger}$, Cristian Caloian ${ }^{1 \dagger}$, Kyle Ellrott ${ }^{3,4}$, J. Christopher Bare ${ }^{2}$, Takafumi N. Yamaguchi ${ }^{1}$, \\ Adam D. Ewing ${ }^{3,5}$, Kathleen E. Houlahan', Thea C. Norman², Adam A. Margolin 2,4,6, Joshua M. Stuart ${ }^{3}$ \\ and Paul C. Boutros ${ }^{1,7,8^{*}}$
}

\begin{abstract}
Background: The clinical sequencing of cancer genomes to personalize therapy is becoming routine across the world. However, concerns over patient re-identification from these data lead to questions about how tightly access should be controlled. It is not thought to be possible to re-identify patients from somatic variant data. However, somatic variant detection pipelines can mistakenly identify germline variants as somatic ones, a process called "germline leakage". The rate of germline leakage across different somatic variant detection pipelines is not wellunderstood, and it is uncertain whether or not somatic variant calls should be considered re-identifiable. To fill this gap, we quantified germline leakage across 259 sets of whole-genome somatic single nucleotide variant (SNVs) predictions made by 21 teams as part of the ICGC-TCGA DREAM Somatic Mutation Calling Challenge.

Results: The median somatic SNV prediction set contained 4325 somatic SNVs and leaked one germline polymorphism. The level of germline leakage was inversely correlated with somatic SNV prediction accuracy and positively correlated with the amount of infiltrating normal cells. The specific germline variants leaked differed by tumour and algorithm. To aid in quantitation and correction of leakage, we created a tool, called GermlineFilter, for use in public-facing somatic SNV databases.

Conclusions: The potential for patient re-identification from leaked germline variants in somatic SNV predictions has led to divergent open data access policies, based on different assessments of the risks. Indeed, a single, wellpublicized re-identification event could reshape public perceptions of the values of genomic data sharing. We find that modern somatic SNV prediction pipelines have low germline-leakage rates, which can be further reduced, especially for cloud-sharing, using pre-filtering software.
\end{abstract}

Keywords: Cancer genomics, Next-generation sequencing, Mutation calling, Germline contamination, Germline leakage, Patient identifiability, Single nucleotide variant, SNV

\section{Background}

The appropriate limits on data sharing remains a contentious issue throughout biomedical research, as shown by recent controversies [1]. Studies such as the Personal Genome Project (PGP) have pioneered open sharing of

\footnotetext{
* Correspondence: paul.boutros@oicr.on.ca

${ }^{\dagger}$ Equal contributors

'Informatics \& Biocomputing Program, Ontario Institute for Cancer Research, 661 University Avenue, Suite 510, Toronto, Ontario M5G 0A3, Canada

${ }^{7}$ Department of Medical Biophysics, University of Toronto, Toronto, Ontario, Canada

Full list of author information is available at the end of the article
}

patient data for biomedical research, while ensuring that enrolled patients consent to risks of identification [2]. In fact, analysis of PGP data has showed that a majority of participants can be linked to a specific named individual [3]. Identifiability is greatly facilitated when researchers release all generated data online - as is standard in some fields [4]. This public, barrier-free release has numerous advantages. It can minimize storage costs, increase data redundancy to reduce the risk of data-loss and maximize data availability and re-use. As a result, it is argued that barrierfree deposition of genomic data in public repositories like 
GEO $[5,6]$ or dbGaP $[7,8]$ promotes collaborative work and maximizes the value of already-funded research [9]. Further, many researchers believe they have an ethical duty to release all data [10].

Nevertheless, there are at least four counter-arguments in favour of a conservative approach to data protection. First, the groups generating the data have uniquely intimate knowledge of it and studies done without their participation can be more prone to errors, although improved documentation of the research process can mitigate this effect [1]. Second, the desire to immediately release data may oppose the desire to explore complex inter-linked questions. The initial report of a dataset may not fully reflect the magnitude of work that goes into generating it, particularly for clinical trials. With immediate data release, the data collectors may find themselves under time constraints, unable to comprehensively exploit the data they produced without competition from subsequent researchers who are able to use the data freely. This effectively disincentivizes the challenging work of dataset creation, producing a situation akin to a tragedy of the commons. Third, the inherent value in large datasets may enable data producers to seek commercialization opportunities by keeping data resources private. Fourth, many studies involve data derived from human subjects that contain revealing and personal information, which is under legal protection [11]. Legislation designed to protect patient privacy, such as the Health Insurance Portability and Accountability Act (HIPAA) [12], the Common Rule [13] and the European Union's General Data Protection Regulation [14] impose harsh financial and professional penalties for violations. As genomic data becomes widely available and techniques for interpreting them improve, de-identification grows increasingly difficult, challenging implementation of barrier-free access that upholds ethical considerations. We focus here on this fourth challenge, or re-identifiability.

Earlier studies have quantified how much DNA information is required to identify individuals. One suggests that as few as 30-80 statistically-independent single nucleotide polymorphisms (SNPs) suffice [15]. Under certain circumstances, small segments of DNA can even be used to recover participants' names by accessing publicly available, commercial genealogy websites [16]. These problems are compounded by deficiencies in techniques used to prevent re-identification: for example, pooling DNA samples does not prevent detection of any individual sequence [17]. More recently, research into information leakage demonstrated how easily patients can be linked back to data from which they previously had been disassociated by correlating seemingly disparate features, namely from phenotypic and genotypic datasets, in what is referred to as a 'linking attack' $[18,19]$.
In cancer research, many studies concentrate on identifying somatic mutations that are induced in the process of tumourigenesis and tumour evolution. Identifying these causative mutations can lead to discovery of novel biomarkers and potential therapeutic targets, making public data release critical for accelerating research. Because these mutations are found in the tumour and not in an individual's germline genome, they do not, by themselves, provide identifying information. Barrier-free release of somatic mutational data can, in theory, occur without compromising patient privacy.

However, tools used to distinguish somatic mutations from germline are imperfect, and sometimes the predicted somatic mutations are in fact germline genetic variants. This "germline leakage" can occur in several ways. Most next-generation sequencing (NGS) base calling algorithms have low error rates [20], including both undetected true variants (false negatives) while some non-existent variants get reported (false positives). These false positives can occur for several reasons, including low coverage (number of reads aligning to a specific position in the genome), which reduces statistical confidence [21]. Even datasets with high total coverage have variable coverage across the genome with particular regions getting sampled at lower rates either through stochastic or structurally biased factors. As a result, sets of somatic variant predictions can be contaminated with germline variants, particularly in the case of single nucleotide variants (SNVs). To account for these errors, some groups filter out any variant seen in a germline database like dbSNP, while others allow only release of mutations in the exome [22]. Still others allow public release of somatic variant predictions from the whole genome [23]. These variations reflect differing views on the likelihoods and risks of germline leakage, and many groups have not yet developed or articulated specific policies.

To help improve our understanding of the magnitude of germline leakage, we analyzed a set of 259 somatic mutation predictions made by 21 groups from around the world on three synthetic tumours during the ICGCTCGA DREAM Somatic Mutation Calling-DNA (SMCDNA) Challenge [24]. We developed a software tool, called GermlineFilter, which can help to quantify and mitigate the risks of germline leakage for publicly available somatic SNV data.

\section{Results}

\section{Gold standards of germline leakage}

We sought to evaluate the extent of germline contamination in contemporary cancer whole-genome sequencing (WGS) datasets, particularly those comprising somatic SNV predictions across the entire genome. To do so, we exploited the synthetic data from the ICGC-TCGA 
DREAM SMC-DNA Challenge [24, 25], which benchmarked somatic SNV predictions using synthetic and real tumour-normal whole-genome pairs. The generation of the synthetic tumours and their properties are fully detailed in Ewing et al. [25]. Briefly, high coverage binary alignment map (BAM) files were obtained from cell lines HCC1143 and HCC1954 [26]. BAMSurgeon [25] was used to randomly 'spike-in' germline mutations into the BAM files. Each file was then split into two: one file representing a synthetic tumour and the other file representing the matched normal. The tumour BAM file was finalized by adding somatic mutations: both SNVs and structural variants. This methodology allows for the creation of a "gold standard" dataset in which the precise locations of germline and somatic variants are known, enabling comprehensive assessment of leaked germline mutations. We focused on the first three synthetic tumours from SMC-DNA, referred to as IS1, IS2 and IS3. These tumours vary in the number of mutations, normal contamination and subclonal complexity (Additional file 1: Table S1) [25]. The synthetic tumours have been available to the public for several years and have thus accumulated a large number of somatic mutation calling results from various submitted methods. Additionally, the organizers ran several widely used algorithms with default settings as a baseline [25]. In total, we evaluated 5,792,868 somatic mutations that included 259 analyses by 21 teams across the three tumours $\left(\mathrm{n}_{\mathrm{IS} 1}=120 ; \mathrm{n}_{\mathrm{IS} 2}=71 ; \mathrm{n}_{\mathrm{IS} 3}=68\right)$.

\section{Assessment of germline leakage}

To quantify germline leakage in submissions to the SMC-DNA tumours, we created a Python program called GermlineFilter, which simultaneously evaluates germline leakage in somatic SNV predictions and filters them in real-time to allow barrier-free access to the final results. The overall process has two steps and the workflow employed by the Challenge administrators has been visualized in Fig. 1. During the initial preprocessing step, a germline caller is run on paired tumour and normal BAM files to generate the germline variant calls. Current germline callers have high accuracy rates which can be attributed to diploidy-based assumptions of normal human tissue, assumptions that do not hold for somatic variants due to a host of issues (e.g. intra-tumour heterogeneity, tissue cellularity, genomic instability). In the following step, each germline SNP is compared against the somatic SNV predictions to be filtered, provided in standard variant call format (VCF), and the matches are identified. Finally, somatic SNV calls can now be filtered, either by rejecting entire submissions that exceed an acceptable level of leakage or by simply removing the calls that match a germline variant. Thus in this mode of execution, a data provider who operates the server can then run GermlineFilter in online mode. This can be used to enable real-time uploads of somatic SNV predictions (as might be done in a benchmarking study), or simply to help prevent inadvertent leakage of germline variants due to erroneous uploads.

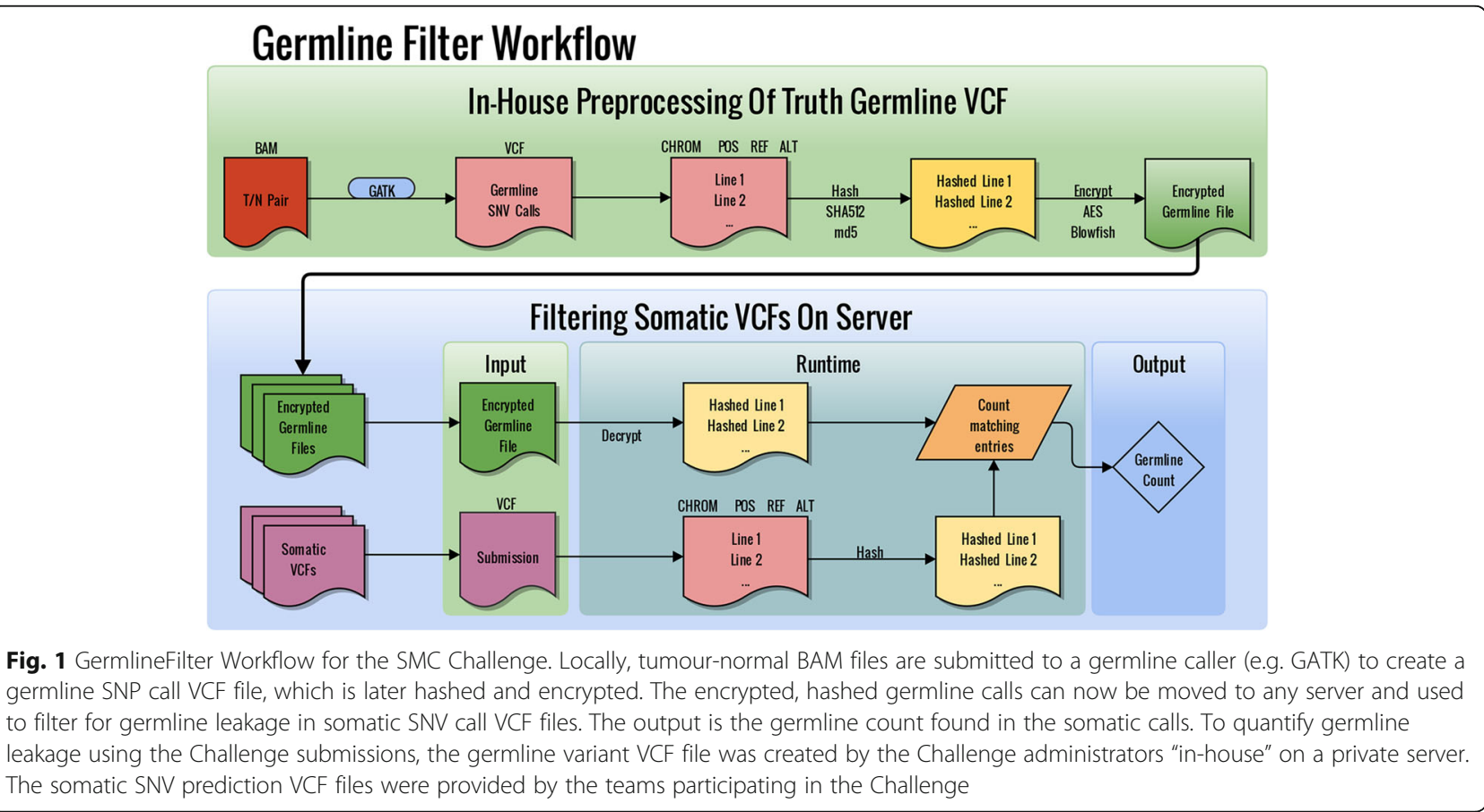




\section{Germline contamination reduces somatic SNV prediction accuracy}

The 259 somatic call VCFs submitted during the IS1, IS2 and IS3 phases of the SMC-DNA challenge contained a median of 4325 SNV calls (averaging 22,366 SNV calls). Each of these was run through GermlineFilter to quantify germline leakage in terms of the number of true germline SNPs misidentified as somatic SNVs. Prediction accuracy for each submission was measured using the $F_{1}$-score (i.e. the harmonic mean of precision and recall) in keeping with the metrics used in the DREAM SMC-DNA challenge.
Germline leakage was highly variable across submissions, ranging from 0 to 45,300 , with a median of 1 per submission. The median leakage rate across tumours ranged from 0 (IS3), to 2 (IS1) and went up as high as 6 (IS2). IS2 contained the highest normal contamination (20\%), suggesting that even low normal contamination can increase germline leakage. For each tumour, we compared germline count to the previously reported $\mathrm{F}_{1}$-scores (Fig. 2a) and found a highly significant negative correlation in each of the three tumours (Spearman's $\rho_{\text {IS1 }}=-0.557, \rho_{\text {IS2 }}=-0.477, \rho_{\text {IS3 }}=-0.410$, Additional file 1: Table S1). For a number of algorithms,

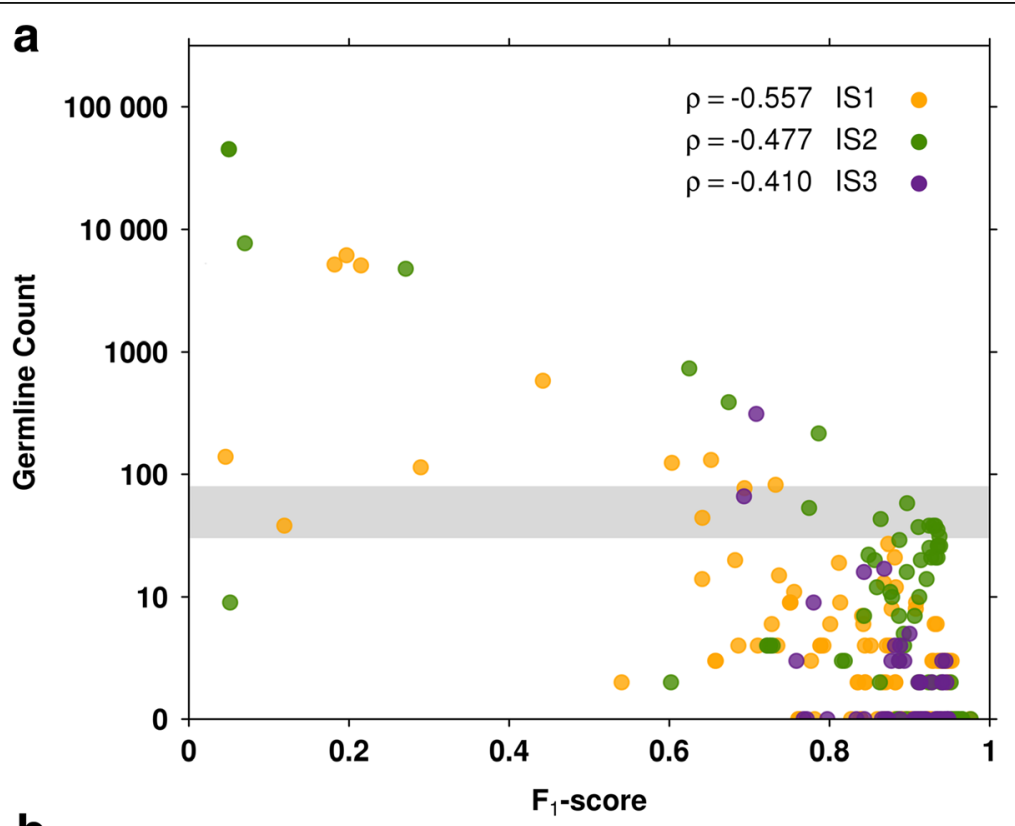

b
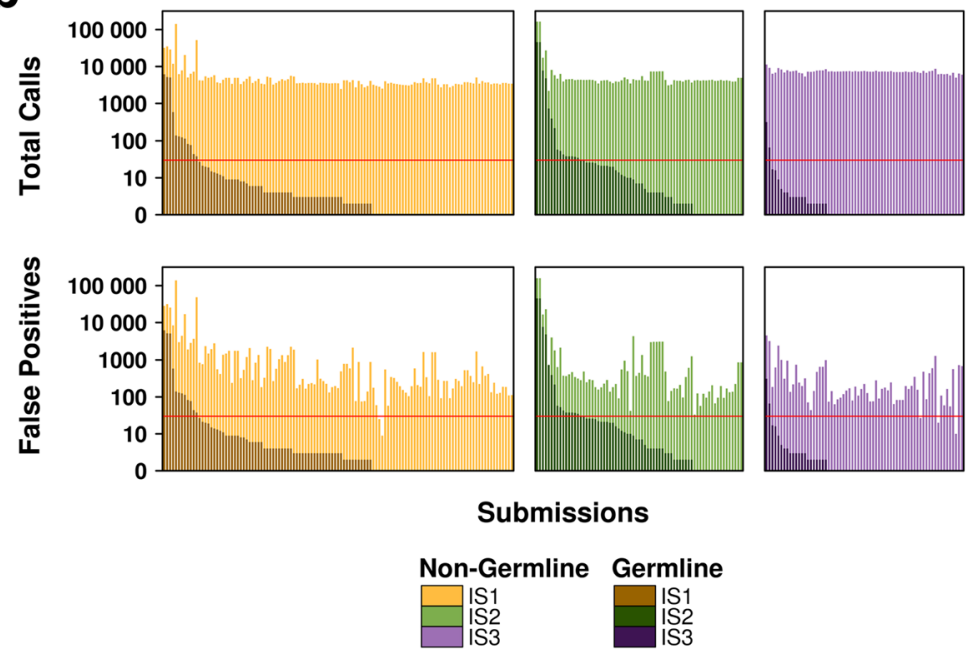

Fig. 2 Assessment of somatic SNV prediction accuracy against germline leakage. a $F_{1}$-scores for each submission are plotted against the germline count (as determined by GermlineFilter). Submissions for different tumours are colour-coded (IS1= orange, IS2 = green, IS3 = purple). The grey area represents 30-80 counts: the minimum number of independent SNPs required to correctly identify a subject, according to Lin et al. [15]. $\mathbf{b}$ Proportions of germline calls as found in total submission calls (upper panel) and in false positive submission calls (lower panel) per tumour. The horizontal red lines indicate the 30 count mark (the lower bound of the 30-80 SNP range mentioned above) 
the germline variants make up a substantial fraction of the total calls, showing an association with the number of false positive calls (Fig. 2b). Thus germline leakage is, as expected, associated with reduced overall accuracy of mutation calling.

\section{Quantifying germline leakage across tumours and between algorithms}

Submissions were further analyzed to determine recurrence of individual germline contaminants across the mutation calling algorithms. For these purposes, only the highest $F_{1}$-score submission from each team was selected, as in the primary report of the somatic SNV data [25]. This was done separately for each tumour, resulting in 15 submissions for IS1, 12 for IS2 and 11 for IS3. A plurality of submissions harboured no germline variants (IS1 $=40.0 \%$; IS2 $=41.7 \%$; IS3 $=45.5 \%$ ), but there was substantial variability, with one submission containing 43 germline SNPs (Additional file 2: Table S2).

Individual leaked germline variants varied significantly across algorithms (Fig. 3). Of the 85 germline variants leaked in the 12 IS2 submissions (all with an $F_{1}>0.863$ ), only five were identified more than once. Similarly, of the 23 germline variants leaked in the 11 IS3 submissions, only two were identified more than once. Leaked variants were distributed uniformly across chromosomes. These data suggest that in modern pipelines, germline leakage rates are low and different variants are leaked by different pipelines.

Due to the voluntary nature of self-reporting Challenge submission details, the specifics on algorithm and data processing techniques employed by the participants were only provided for a minority of the submissions [25]. However, this information is available for submissions created by the Challenge administrators, where several popular SNV calling algorithms were selected and run with default parameters on tumours IS1 and IS2. Germline leakage was quantified for the submissions generated using SNV callers Strelka [27], MuTect [28] and VarScan [29]. Strelka had both the highest-scoring performance for tumours IS1 $\left(\mathrm{F}_{1}\right.$-score $\left.=0.871\right)$ and IS2 $\left(\mathrm{F}_{1}\right.$-score $\left.=0.887\right)$ and very low germline leakage in the somatic variant predictions (IS1 = 3; IS2 =6). However, despite worse overall performance, MuTect-derived somatic predictions contained even fewer germline leaks with 2 leaks in IS1

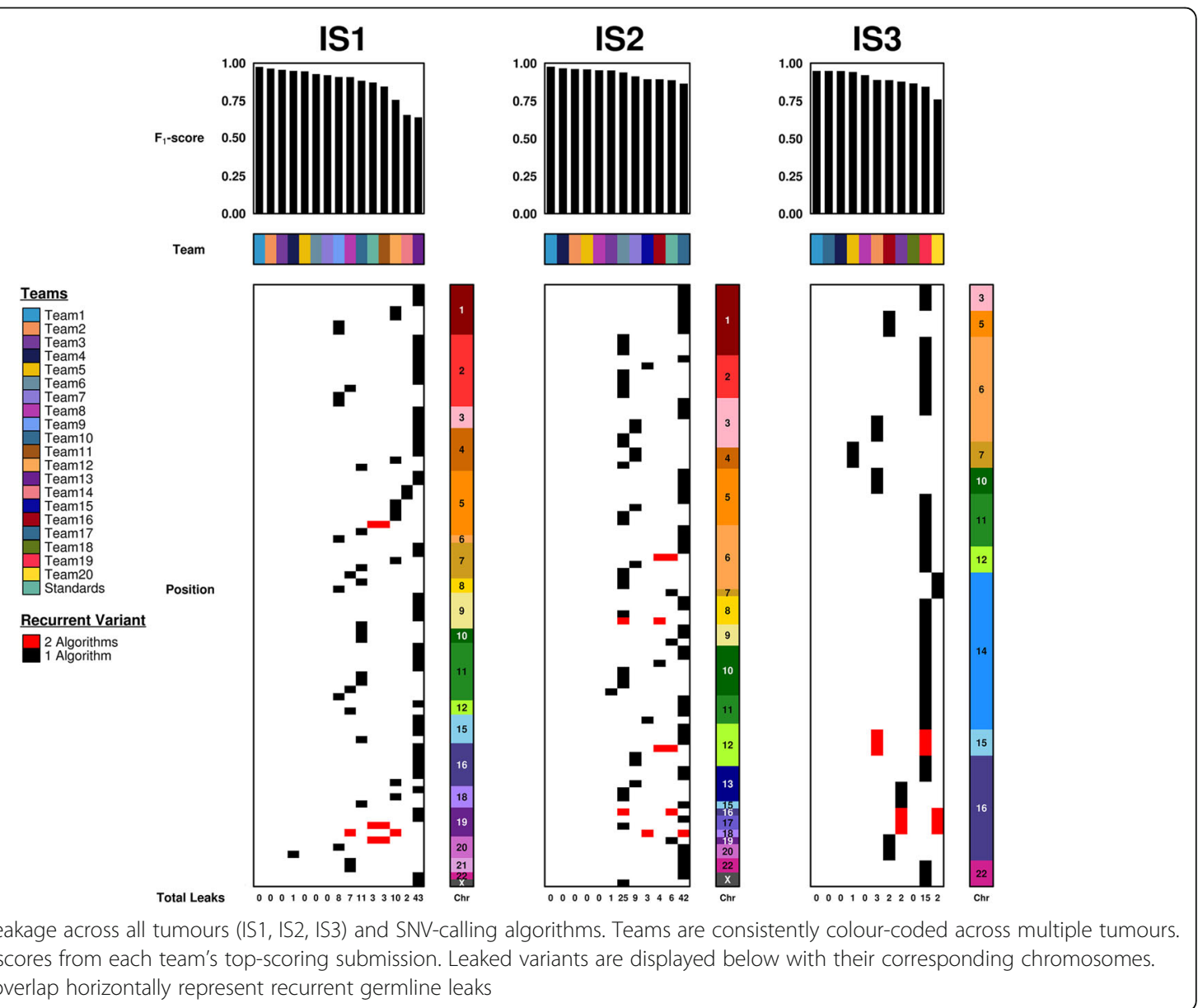


results and 3 leaks in IS2 results. Importantly none of these analyses used post-filtering, so these reflect the true germline leakage rates of the algorithms in isolate, at their state of development in 2014-2015. This thus provides an upper-bound on the leakage rate of even relatively simple somatic detection pipelines.

To complement these findings, we analyzed reports for the top-scoring submission from each of the three tumours. Interestingly, each of these prediction sets was generated using MuTect and all three contained zero germline leaks (Fig. 3). This suggests that parameter optimization can substantially improve overall caller performance while further minimizing germline leakage.

In addition to the spiked-in mutations, common SNP sites were also analyzed. The Exome Aggregation Consortium (ExAC) has produced a library of variant sites seen across 60,706 individuals [30]. These sites represent locations where samples commonly deviate from the reference. Due to the very large number of individuals represented, this set of SNP sites is often used as a filter of possible germline variant sites. ExAC provides $~ 9.3$ million potential common SNP sites, much more than the thousands of spiked-in mutations. The number of false positive calls using ExAC as a filter remained very low (medians: IS $1=2$; IS2 $=3$; IS $3=1.5$ ). As these sites are publicly available and known to be common for SNPs, most modern somatic calling pipelines can directly incorporate this information into their filtering strategy.

\section{Discussion}

Barrier-free access to genomic data can expand its utility, maximizing investments in research funding, enabling citizen-scientists and facilitating collaboration. Strong barriers to access can limit these positive consequences of large investments in dataset generation. Indeed, even when data is made available through protected databases, the processes to gain access can be time-consuming, advantaging labs or institutions that have resources dedicated to gaining and maintaining data-access authorizations. Accessibility can be skewed by variability in the standards, knowledge and impartiality of data access committees that authorize use of controlled data [31, 32].

We quantified the amount of leakage in three comprehensively studied tumours used in a crowd-sourced prediction benchmarking challenge. While some submissions showed large amounts of germline leakage, the median submission leaked only one germline SNP, and indeed the top three teams for each tumour leaked none. Given that the SMC-DNA Challenge was run in 20142015 and that detection pipelines and the quality of genomic data have improved further since, it appears that modern optimized variant-calling pipelines leak an insignificant number of germline variants on many tumours, well below the 30-80 independent SNP range needed for re-identification [15].

However, several caveats must be evaluated when considering barrier-free access to whole-genome somatic SNV predictions. First, the data we evaluated only included three tumours, and further evaluations on larger numbers with a range of cellularities will be critical to generalize these conclusions. Additionally, while we considered the amount of germline leakage in tumors with different subclonal complexities, we did not investigate whether germline leakage is more likely in genomic regions with specific tumour characteristics (e.g. mutational hotspots, trinucleotide context, subclonality, copy number alterations, loss of heterozygosity, etc.). On-going work from the ICGC Pan-Cancer Analysis of Whole Genomes (PCAWG) may provide the data necessary to address this. Second, genomic alterations other than nuclear SNVs (e.g. germline copy number variants and mitochondrial polymorphisms) may provide information contributing to identifiability. Third, while most individual pipelines leaked few variants, aggregating multiple pipelines could increase the information content: the union of variants across all 12 pipelines from IS2 contain 85 leaked SNPs, potentially providing sufficient information for reidentification [15]. Since ensemble calling generally adopts a 'majority rules' approach [33], which would remove most germline variants due to low recurrence, this is most relevant in cases of malicious intent. Finally, there is some inherent trade-off to the use of GermlineFilter as a software solution to help mitigate leakage: it will inevitably slightly increase the false-negative rate of somatic detection, by about $0.1 \%$ in our dataset. Given the challenges with sharing genomic data to date and the need to maximize data openness, this may be an acceptable tradeoff for almost all biological questions.

\section{Conclusions}

Taken together, our findings suggest that germline contamination in somatic SNV calling is relatively rare, and supports additional consideration of barrier-free access to these data. Re-identification risks can be substantially reduced by incorporating automated checks into the data release process, designed to identify germline leakage and remove these prior to data release. GermlineFilter provides a convenient and secure way to monitor leakage by individual algorithms, and may be useful as a front-end to cloud-based SNV databases to quantify and minimize risk in real-time.

\section{Methods}

\section{Software}

GermlineFilter works in an encrypted fashion, allowing its use on a public server. The software is executed in 
two steps (Fig. 1). For the first step, performed offline, a VCF file containing germline calls is generated using paired tumour and normal BAM files. For each germline SNP in the VCF file, the chromosome, position, reference base and alternate base are extracted. This information is hashed and written to a file that is then encrypted. It is this encrypted file of hashes rather than the actual variants that is then transferred to the server. It is technically possible to reveal the actual germline variants if their hashes are successfully matched with hashes of known variants. As such, the encryption serves as an additional security measure. For the next step, online somatic VCF filtering is performed. At runtime, the truth germline VCF is decrypted in memory and the somatic VCF undergoes preprocessing and hashing. Finally, an in-memory comparison of hashes is done and the number of matches is returned. At no point are the decrypted germline variant hashes stored on the server. GermlineFilter can spawn multiple instances to process multiple germline VCFs for different tumours or multiple somatic VCFs for a single tumour. The user chooses the encryption and hashing protocols, with strong default settings in place to help minimize risks such as hash collisions. The user also has the option to specify alternative germline call sets, such as a list of all dbSNP entries, although these would elevate the false-negative rate by removing true somatic mutations. Another feature for local use allows the user to obtain a list of the actual positions of the germline leaks within the somatic VCF. This list can be used to filter out the germline mutations in preparation for publication.

The GermlineFilter software package was written in Python 2.7 and it is supported for Unix and Linux platforms. The encryption and hashing is done using the PyCrypto v2.6.1 Python module. The tool currently supports two encryption protocols - AES (default) and Blowfish, as well as two hashing protocols - SHA512 (default) and $m d 5$, selected for their security and broad usage. GermlineFilter v1.2 is the stable version and it is available for download at: https://pypi.python.org/pypi/ GermlineFilter. Alternatively, it can be installed via pip install GermlineFilter.

\section{Data}

The analysis data was taken from Ewing et al. [25] and it consists of the first three publicly available in silico datasets from the ICGC-TCGA DREAM Somatic Mutation Calling Challenge and their corresponding SNV submissions from the challenge participants. The truth germline calls were generated using GATK HaplotypeCaller v3.3. A description of the synthetic tumour data and a summary of participating teams and their submissions can be found in Additional file 1: Table S1. All challenge submissions and their scores are listed in Additional file 2: Table S2.

For each of the 259 submissions we calculated: precision (the fraction of submitted calls that are true somatic SNVs), recall (the fraction of true somatic SNVs that are identified by the caller) and the $F_{1}$ score (the harmonic mean of precision and recall), as previously reported [25]. The $F_{1}$-score was selected to be the accuracy metric as it does not rely on true negative information which, given the nature of somatic variant calling on whole genome sequencing data, would overwhelm alternative scoring metrics such as specificity (the fraction of non-SNV bases that are correctly identified as such by the caller).

Each tumour's germline calls were encrypted separately using default methods: AES for encryption and SHA512 for hashing. Somatic calls from all challenge submissions were filtered against their corresponding tumour's encrypted germline calls. For a somatic SNV call to be designated a germline leak, it exactly matched a germline variant at the chromosome, position, reference allele and alternate allele.

The resulting germline leak counts were compared to $\mathrm{F}_{1}$-scores using Spearman correlation. The best team submissions per tumour were selected to look at leaked germline variant recurrence across tumours and mutation callers. Best submissions were defined as having the highest $\mathrm{F}_{1}$-score.

\section{Visualization}

All data figures were created using custom $R$ scripts executed in the $\mathrm{R}$ statistical environment (v3.2.3) using the BPG (v5.6.8) package [34].

\section{Additional files}

Additional file 1: Table S1. Tumour information from each tumour challenge (IS1, IS2, IS3). This includes information on in silico tumour construction, composition, and a summary of participating teams and their challenge submissions. (XLS $12 \mathrm{~kb}$ )

Additional file 2: Table S2. Contains the following information for every challenge submission: tumour, submission ID, precision, recall, $F_{1}$ score, the number of germline variants leaked and whether it was a Challenge administrator submission. (XLS 39 kb)

\section{Abbreviations}

BAM: Binary alignment map; DREAM: Dialogue on reverse-engineering assessment and methods; GATK: Genome analysis toolkit; HIPAA: Health information portability and accountability act; ICGC: International cancer genome consortium; NGS: Next-generation sequencing; PGP: Personal genome project; SMC: Somatic mutation calling; SNP: Single nucleotide polymorphism; SNV: Single nucleotide variant; TCGA: The cancer genome atlas; VCF: Variant call format

\section{Acknowledgements}

The authors thank all members of the Boutros lab and all ICGC-TCGA DREAM Somatic Mutation Calling Challenge Participants for their support and thoughtful commentary. 


\section{Funding}

This study was conducted with the support of the Ontario Institute for Cancer Research to P.C.B. through funding provided by the Government of Ontario. This work was supported by Prostate Cancer Canada and is proudly funded by the Movember Foundation - Grant \#RS2014-01. This project was supported by Genome Canada through a Large-Scale Applied Project contract to P.C.B., S.P. Shah and R.D. Morin. This work was supported by the Discovery Frontiers: Advancing Big Data Science in Genomics Research program, which is jointly funded by the Natural Sciences and Engineering Research Council (NSERC) of Canada, the Canadian Institutes of Health Research (ClHR), Genome Canada, and the Canada Foundation for Innovation (CFI). P.C.B. was supported by a Terry Fox Research Institute New Investigator Award and a CIHR New Investigator Award. The following NIH grants supported this work: R01-CA180778 (J.M.S.), U24-CA143858 (J.M.S.), and U54HG007990 (A.A.M.). The authors thank Google Inc. (in particular N. Deflaux) and Annai Biosystems (in particular D. Maltbie and F. De La Vega) for their ongoing support of the ICGC-TCGA DREAM Somatic Mutation Calling Challenge.

\section{Availability of data and materials}

The datasets supporting the conclusions of this article are available on Synapse (syn312572) at: https://www.synapse.org/\#!Synapse:syn312572/wiki/ 61509, and in the Supplementary of Ewing et al. [25]. The main GermlineFilter project page is at: https://labs.oicr.on.ca/boutros-lab/software/ germlinefilter, and the source-code is freely available at: https://pypi.python.org/pypi/GermlineFilter/1.2.

\section{Authors' contributions}

ADE, AAM, JMS and PCB initiated the project. CC created GermlineFilter and performed validation studies. KE, CC, JCB, TCN, AAM, JMS and PCB created the ICGC-TCGA DREAM Somatic Mutation Calling Challenge. DHS, CC, TNY, $\mathrm{KEH}$ and ADE created datasets and analyzed submission data. Research was supervised by AAM, JMS and PCB. The first draft of the manuscript was written by DHS, and approved by all authors.

\section{Ethics approval and consent to participate}

Not applicable

\section{Consent for publication}

Not applicable

\section{Competing interests}

All authors declare that they have no competing interests.

\section{Publisher's Note}

Springer Nature remains neutral with regard to jurisdictional claims in published maps and institutional affiliations.

\section{Author details}

${ }^{1}$ Informatics \& Biocomputing Program, Ontario Institute for Cancer Research, 661 University Avenue, Suite 510, Toronto, Ontario M5G 0A3, Canada. ${ }^{2}$ Sage Bionetworks, Seattle, WA, USA. 'Department of Biomolecular Engineering, University of California, Santa Cruz, Santa Cruz, CA, USA. ${ }^{4}$ Computational Biology Program, Oregon Health \& Science University, Portland, OR, USA. ${ }^{5}$ Mater Research Institute, University of Queensland, Woolloongabba, Queensland, Australia. ${ }^{6}$ Department of Biomedical Engineering, Oregon Health \& Science University, Portland, OR, USA. ${ }^{7}$ Department of Medical Biophysics, University of Toronto, Toronto, Ontario, Canada. ${ }^{8}$ Department of Pharmacology \& Toxicology, University of Toronto, Toronto, Ontario, Canada.

Received: 16 October 2017 Accepted: 24 January 2018

Published online: 31 January 2018

\section{References}

1. Longo DL, Drazen JM. Data Sharing. N Engl J Med. 2016;374:276-7.

2. Personal Genome Project. Harvard Medical School, Boston. 2017. http:// www.personalgenomes.org. Accessed 12 Oct 2017

3. Sweeney L, Abu A, Winn J. Identifying participants in the personal genome project by name (a re-identification experiment). CoRR. 2013;abs/1304.7605: http://arxiv.org/abs/1304.7605.
4. Toronto International Data Release Workshop Authors, Birney E, Hudson TJ, Green ED, Gunter C, Eddy S, Rogers J, Harris JR, Ehrlich SD, Apweiler R, Austin CP, Berglund L, Bobrow M, Bountra C, Brookes AJ, Cambon-Thomsen A, Carter NP, Chisholm RL, Contreras JL, Cooke RM, Crosby WL, Dewar K, Durbin R, Dyke SO, Ecker JR, El Emam K, Feuk L, Gabriel SB, Gallacher J, Gelbart WM, Granell A, Guarner F, Hubbard T, Jackson SA, Jennings JL, Joly Y, Jones SM, Kaye J, Kennedy KL, Knoppers BM, Kyrpides NC, Lowrance WW, Luo J, JJ MK, Martín-Rivera L, WR MC, JD MP, Miller L, Miller W, Moerman D, Mooser V, Morton CC, Ostell JM, Ouellette BF, Parkhill J, Raina PS, Rawlings C, Scherer SE, Scherer SW, Schofield PN, Sensen CW, Stodden VC, Sussman MR, Tanaka T, Thornton J, Tsunoda T, Valle D, Vuorio El, Walker NM, Wallace S, Weinstock G, Whitman WB, Worley KC, Wu C, Wu J, Yu J. Prepublication data sharing. Nature. 2009;461:168-70.

5. Edgar R, Domrachev M, Lash AE. Gene expression omnibus: NCBI gene expression and hybridization array data repository. Nucleic Acids Res. 2002;30:207-10

6. Barrett T, Wilhite SE, Ledoux P, Evangelista C, Kim IF, Tomashevsky M, Marshall KA, Phillippy KH, Sherman PM, Holko M, Yefanov A, Lee H, Zhang N, Robertson CL, Serova N, Davis S, Soboleva A. NCBI GEO: archive for functional genomics data sets-update. Nucleic Acids Res. 2013;41:D991-5.

7. Mailman MD, Feolo M, Jin Y, Kimura M, Tryka K, Bagoutdinov R, Hao L, Kiang A, Paschall J, Phan L, Popova N, Pretel S, Ziyabari L, Lee M, Shao Y, Wang ZY, Sirotkin K, Ward M, Kholodov M, Zbicz K, Beck J, Kimelman M, Shevelev S, Preuss D, Yaschenko E, Graeff A, Ostell J, Sherry ST. The NCBI $\mathrm{dbGaP}$ database of genotypes and phenotypes. Nat Genet. 2007;39:1181-6.

8. Tryka KA, Hao L, Sturcke A, Jin Y, Wang ZY, Ziyabari L, Lee M, Popova N, Sharopova N, Kimura M, Feolo M. NCBI's database of genotypes and phenotypes: dbGaP. Nucleic Acids Res. 2014;42:D975-9.

9. Rodriguez LL, Brooks DB, Greenberg JH, Green ED. Research ethics. The complexities of genomic identifiability. Science. 2013;339:275-6.

10. Lolkema MP, Gadellaa-van Hooijdonk CG, Bredenoord AL, Kapitein P, Roach N, Cuppen E, Knoers NV, Voest EE. Ethical, legal, and counseling challenges surrounding the return of genetic results in oncology. J Clin Oncol. 2013:31, 1842-1838.

11. Lowrance WW, Collins FS. Ethics. Identifiability in genomic research. Science. 2007;317:600-2.

12. U.S. Department of Health \& Human Services: Health information privacy. http://www.hhs.gov/hipaa/.

13. U.S. Department of Health \& Human Services: Federal Policy for the protection of human subjects ('Common Rule'). https://www.hhs.gov/ohrp/ regulations-and-policy/regulations/common-rule/.

14. European Commission: Justice: protection of personal data. https://ec. europa.eu/info/strategy/justice-and-fundamental-rights/data-protection_en.

15. Lin Z, Owen AB, Altman RB. Genetics. Gen Res Hum Subj Privacy Sci. 2004; 305:183.

16. Gymrek M, McGuire AL, Golan D, Halperin E, Erlich Y. Identifying personal genomes by surname inference. Science. 2013;399:321-4.

17. Homer N, Szelinger S, Redman M, Duggan D, Tembe W, Muehling J, Pearson JV, Stephan DA, Nelson SF, Craig DW. Resolving individuals contributing trace amounts of DNA to highly complex mixtures using highdensity SNP genotyping microarrays. PLoS Genet. 2008;4:e1000167.

18. Harmanci A, Gerstein M. Quantification of private information leakage from phenotype-genotype data: linking attacks. Nat Methods. 2016;13:251-6.

19. Craig DW. Understanding the links between privacy and public data sharing. Nat Methods. 2016;13:211-2.

20. Harismendy O, Ng PC, Strausberg RL, Wang X, Stockwell TB, Beeson KY, Schork NJ, Murray SS, Topol EJ, Levy S, Frazer KA. Evaluation of next generation sequencing platforms for population targeted sequencing studies. Genome Biol. 2009;10:R32.

21. Meyerson M, Gabriel S, Getz G. Advances in understanding cancer genomes through second-generation sequencing. Nat Rev Genet. 2010;11:685-96.

22. The Cancer Genome Atlas Data Portal. Data levels and data types: DNA sequencing. https://tcga-data.nci.nih.gov/docs/publications/tcga/datatype. html. Accessed 29 Jan 2016.

23. International Cancer Genome Consortium. Goals, structure, policies \& guidelines. 2008. https://icgc.org/files/icgc/ICGC_April_29_2008_en.pdf. Accessed 01 Feb 2016

24. Boutros PC, Ewing AD, Ellrott K, Norman TC, Dang KK, Hu Y, Kellen MR, Suver C, Bare JC, Stein LD, Spellman PT, Stolovitzky G, Friend SH, Margolin AA, Stuart JM. Global optimization of somatic variant identification in cancer genomes with a global community challenge. Nat Genet. 2014;46:318-9. 
25. Ewing AD, Houlahan KE, Hu Y, Ellrott K, Caloian C, Yamaguchi TN, Bare JC, P'ng C, Waggott D, Sabelnykova VY; ICGC-TCGA DREAM Somatic Mutation Calling Challenge participants, Kellen MR, Norman TC, Haussler D, Friend SH, Stolovitzky G, Margolin AA, Stuart JM, Boutros PC. Combining tumor genome simulation with crowdsourcing to benchmark somatic singlenucleotide-variant detection. Nat Methods 2015;12:623-630.

26. Gazdar AF, Kurvari V, Virmani A, Gollahon L, Sakaguchi M, Westerfield M, Kodagoda D, Stasny V, Cunningham HT, Wistuba II, Tomlinson G, Tonk V, Ashfaq R, Leitch AM, Minna JD, Shay JW. Characterization of paired tumor and non-tumor cell lines established from patients with breast cancer. Int J Cancer. 1998;78:766-74.

27. Saunders CT, Wong WS, Swamy S, Becq J, Murray LJ, Cheetham RK. Strelka: accurate somatic small-variant calling from sequenced tumor-normal sample pairs. Bioinformatics. 2012;28(14):1811-7.

28. Cibulskis K, Lawrence MS, Carter SL, Sivachenko A, Jaffe D, Sougnez C, Gabriel S, Meyerson M, Lander ES, Getz G. Sensitive detection of somatic point mutations in impure and heterogeneous cancer samples. Nat Biotechnol. 2013;31(3):213-9.

29. Koboldt DC, Zhang Q, Larson DE, Shen D, McLellan MD, Lin L, Miller CA, Mardis ER, Ding L, Wilson RK. VarScan2: somatic mutation and copy number alteration discovery in cancer by exome sequencing. Genome Res. 2012; 22(3):568-76.

30. Exome Aggregation Consortium, Lek M, Karczewski KJ, Minikel EV, Samocha KE, Banks E, Fennell T, O'Donnell-Luria AH, Ware JS, Hill AJ, Cummings BB, Tukiainen T, Birnbaum DP, Kosmicki JA, Duncan LE, Estrada K, Zhao F, Zou J, Pierce-Hoffman E, Berghout J, Cooper DN, Deflaux N, DePristo M, Do R, Flannick J, Fromer M, Gauthier L, Goldstein J, Gupta N, Howrigan D, Kiezun A, Kurki Ml, Moonshine AL, Natarajan P, Orozco L, Peloso GM, Poplin R, Rivas MA, Ruano-Rubio V, Rose SA, Ruderfer DM, Shakir K, Stenson PD, Stevens C, Thomas BP, Tiao G, Tusie-Luna MT, Weisburd B, Won HH, Yu D, Altshuler DM, Ardissino D, Boehnke M, Danesh J, Donnelly S, Elosua R, Florez JC, Gabriel SB, Getz G, Glatt SJ, Hultman CM, Kathiresan S, Laakso M, McCarroll S, McCarthy MI, McGovern D, McPherson R, Neale BM, Palotie A, Purcell SM, Saleheen D, Scharf JM, Sklar P, Sullivan PF, Tuomilehto J, Tsuang MT, Watkins HC, Wilson JG, Daly MJ, MacArthur DG. Analysis of protein-coding genetic variation in 60,706 humans. bioRxiv 2015; doi: https://doi.org/10. 1101/030338

31. Shabani M, Dyke SOM, Joly Y, Borry P. Controlled access under review: improving the governance of genomic data access. PLoS Biol. 2015;13: e1002339.

32. Joly Y, de Vries-Seguin E, Chalmers D, Ouellette BFF, Yamada J, Bobrow M. Knoppers BM for the ICGC data access compliance office and the ICGC international data access committee. Analysis of five years of controlled access and data sharing compliance at the international cancer genome consortium. Nat Genet. 2016;48:224-5.

33. Sage Bionetworks. TCGA unified ensemble "MC3" call set. 2016. https:// www.synapse.org/\#!!Synapse:syn7214402/wiki/. Accessed 11 Oct 2017.

34. P'ng C, Green J, Chong LC, Waggott D, Prokopec SD, Shamsi M, Nguyen F, Mak DYF, Lam F, Albuquerque MA, Wu Y, Jung EH, Starmans MHW, ChanSeng-Yue MA, Yao CQ, Liang B, Lalonde E, Haider S, Simone NA, Sendorek D, Chu KC, Moon NC, Fox NS, Grzadkowski MR, Harding NJ, Fung C, Murdoch AR, Houlahan KE, Wang J, Garcia DR, de Borja R, Sun RX, Lin X, Chen GM, Lu A, Shiah Y-J, Zia A, Kearns R, Boutros P. BPG: seamless, automated and interactive visualization of scientific data. bioRxiv 2017; doi: https://doi.org/10.1101/156067.

\section{Submit your next manuscript to BioMed Central and we will help you at every step:}

- We accept pre-submission inquiries

- Our selector tool helps you to find the most relevant journal

- We provide round the clock customer support

- Convenient online submission

- Thorough peer review

- Inclusion in PubMed and all major indexing services

- Maximum visibility for your research

Submit your manuscript at www.biomedcentral.com/submit 